\title{
Intervention of Dream Quality in the Appearance of Concomitant Symptoms in Hospitalized Patients
}

\author{
Gabriel Miranda Nava ${ }^{1 *}$ and Néstor Javier Escoto Leyva ${ }^{2}$ \\ ${ }^{1}$ Head of the Neurology service, Regional Military Hospital of specialties of Guadalajara, Mexico \\ ${ }^{2}$ Undergraduate Internal Medicine, Mexico
}

*Corresponding author: Gabriel Miranda Nava, Head of the Neurology service, Regional Military Hospital of specialties of Guadalajara, Mexico

\begin{tabular}{|c|c|}
\hline ARTICLE INFO & ABSTRACT \\
\hline Received: 㐩 June 11, 2019 & \multirow{3}{*}{$\begin{array}{l}\text { The present work allows us to observe the quality of sleep and the respect of cir- } \\
\text { cadian cycles as very important factors in the development of multiple diseases and in } \\
\text { various specialties, which affects therapeutic methods, both in outpatients and in hos- } \\
\text { pitalized patients and the variety of symptoms that can be observed, through a method } \\
\text { of surveys in a practical way, with the main objective of contributing in the awareness to } \\
\text { treating physicians about the importance of sleep hygiene measures and their improve- } \\
\text { ment in the quality of life of the patients. This document aims to fulfill the quality of sleep } \\
\text { and the circadian respect cycles as important factors in the development of multiple } \\
\text { diseases in various specialties, which affects both methods and in hospitalized ones, and } \\
\text { the variety of symptoms, that can be observed, through a practical survey method, with } \\
\text { the main objective of contributing to the awareness of treating physicians on the impor- } \\
\text { tance of sleep hygiene measures, and improving patients quality life. }\end{array}$} \\
\hline Published: June 20, 2019 & \\
\hline $\begin{array}{l}\text { tation: Gabriel Miranda N, Néstor } \\
\text { vier Escoto L. Intervention of Dream } \\
\text { dality in the Appearance of Concomi- } \\
\text { nt Symptoms in Hospitalized Patients. } \\
\text { omed J Sci \& Tech Res 19(1)-2019. } \\
\text { STR. MS.ID.003232. }\end{array}$ & \\
\hline
\end{tabular}

\section{Introduction}

When trying to define the dream it is inevitable to differentiate the physiological characteristics in the good quality of sleep depending on the species, where it is a little simpler to enumerate the behavioral characteristics that are associated with the aspect of the dream and try to define it properly, where it can be associated with immobility and muscle relaxation, it is a reversible event (which differentiates it from other pathological states such as stupor and coma), decreased consciousness and reactivity to external stimuli and finally during sleep individuals acquire a stereotyped posture [1]. The absence of sleep induces different behavioral and physiological alterations in addition to generating a cumulative sleep debt that eventually must be recovered. A tool that has been of vital importance for the study of sleep physiology is the electroencephalogram (EEG), which is the graphic and digital representation of the oscillations that show the electrical activity of the brain, to be recorded by electrodes placed in different regions of the head. During alert states while keeping the eyes closed on the EEG, oscillations of electrical activity are observed that are usually between 8-13 cycles per second, mainly in occipital regions (alpha rhythm) [2]. Within the dream, characteristic changes of brain activity occur. which is the basis for dividing the dream into several phases, where it is usually divided into two phases: without rapid eye movements, and with rapid eye movements

\section{Sleep NOT MOR}

Phase N1 corresponds to drowsiness or the onset of light sleep, light muscular jerks are usually observed (acute vertex waves), phase N2 appear specific patterns of brain activity, called sleep spindle and complex $\mathrm{k}$, heart rate and respiratory begins to decrease gradually, phase N3 or slow wave sleep is the deepest phase observed in the EEG very slow frequency activity $(<2 \mathrm{~Hz})$.

\section{MOR Sleep}

Now it is called the R phase and is characterized by the presence of rapid eye movements, physically all muscle tone decreases [35]. A young adult spends approximately 70-100 min in non-MOR sleep, which can last between 5-30 min and this cycle is repeated every hour and a half throughout the night, therefore throughout the night they can be presented normally between 4 and 6 MOR sleep cycles. 


\section{Sleep Hygiene}

Sleep hygiene measures are a series of recommendations about desirable behaviors and habits, as well as modifications of environmental conditions and other related factors, aimed at improving the sleep quality of people who already suffer from a sleep disorder such as insomnia, or that can be used as measures to prevent a sleep disturbance [6,7]. Although there is no global consensus about what these sleep hygiene measures should be and some of these sleep hygiene measures are transplanted with some forms of non-pharmacological behavioral treatment we can generally consider (avoid prolonged naps> 1hr., lie down to sleep at the same time every day, wake up at the same time all day, sleep in a comfortable bed, sleep in a room with low lighting and little ambient noise).

\section{Epidemiology}

It is estimated that, in our country, around $45 \%$ of the adult population has poor sleep quality. The above is reflected in the difficulty that people have to get up, as well as in constant drowsiness and fatigue during the first hours of the morning.

\section{Method}

The present study uses the referential method as a basis, mentioning descriptive surveys, and testing the Pittsburgh quality of sleep scale in which 20 hospitalized patients are evaluated in the Regional Military Hospital of Specialties of Guadalajara, Jalisco, assessing the level of quality of sleep and what concomitant symptoms are added by poor sleep hygiene, February-May trial period [7-10].

\section{Justification}

The present investigation will focus on studying the hygiene habits based on the quality of sleep in hospitalized patients, since due to work stress or in hospital instances it has been seen that patients have directly modified their sleep habits, worsening the quality of life and By delaying the recovery in the hospital by increasing concomitant symptoms, we propose then to investigate the evolution of the pathological patterns and their aggregate symptoms; the reasons that led us to investigate the quality of sleep in hospitalized patients are due to the increase in discomfort or somnolence data reported by patients after staying more than 2 days in hospital. We think that by means of the detailed analysis we will be able to make the health personnel aware to improve the hygiene of the sleep thus helping the quick recovery without the appearance of concomitant symptoms in the patients who are hospitalized.

\section{Goals}

a) Clinically observe the behavior of hospitalized patients and their quality in sleep hygiene.

b) Intervening and preventing concomitant pathologies that are associated with poor hygienic sleep habits.

c) Improve the quality of life of hospitalized patients.

d) Awareness of the application of hygiene or good sleep habits to improve the quality of life.

e) Locate key events or outstanding problems in the quality of sleep.

\section{Statistical Population}

Sampling was done in which people of both sexes were chosen, who underwent a descriptive evaluation through direct surveys, where a sample of 20 people with characteristics with a simple random election type was collected, making a list of participants hospitalized to form the sample to be studied by means of questionnaires.

\section{Results}

A random sample is taken to carry out descriptive direct surveys at the Regional Military Hospital of Guadalajara, Jalisco, where 14 people of the female sex and 6 of the male sex are taken, having a percentage of $70 \%$ female and $30 \%$ male. Regarding age, there is an ambiguous percentage where it is observed that $30 \%$ corresponds to people between 21 and 30 years of age following proportionally $15 \%$ of patients aged 31 to 73 years. In the marital status $74 \%$ of the respondents are married, $11 \%$ are single, and $15 \%$ are proportionally divorced, widowed and in free union (Figures 1-4). The descriptive surveys did not take into account the situations of right-of-way in the institution where the majority of those interviewed or those who were interviewed were the rightful claimants in the asset, having the majority percentage with $60 \%$ followed by military personnel in the asset where it was noted that most of the concomitant symptoms corresponded to anxiety and headache (Figure 5). Among the key questions that are characterized in the scale of Pittsburgh manifested bedtime where literally $50 \%$ of the patients interviewed reiterated to sleep at around 10:00 pm and 11:00 pm, while the rest were distributed with $25 \%$ who go to bed regularly from $08: 00 \mathrm{pm}$ to $09: 00 \mathrm{pm}$ and the other $25 \%$ after 12:00 pm (Figures 6-10).

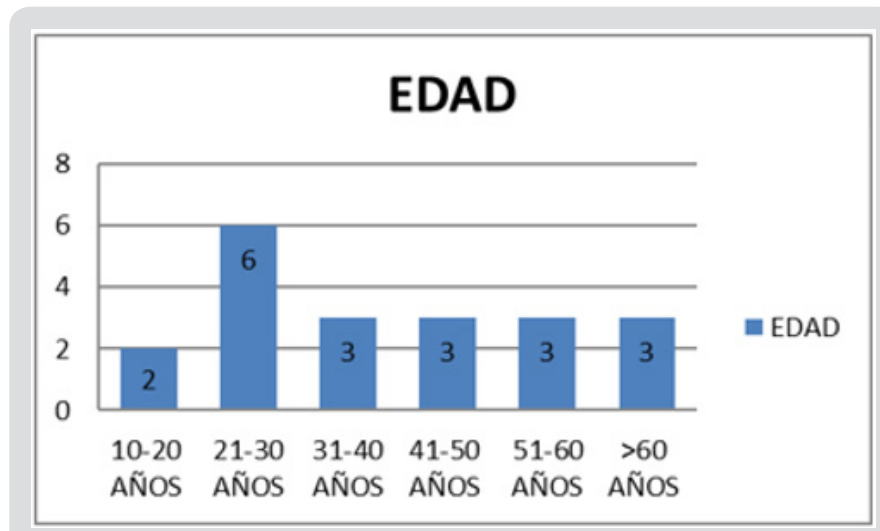

Figure 1: Age of the patients in the sample. 


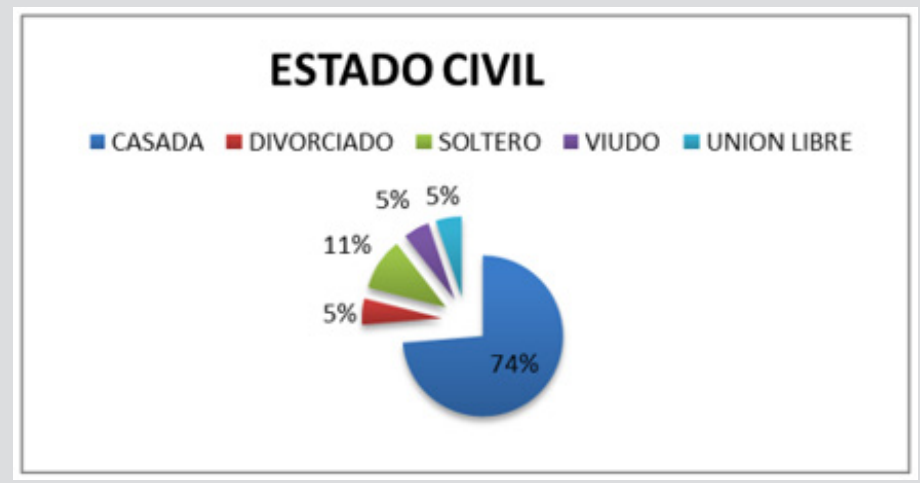

Figure 2: Civil Status.

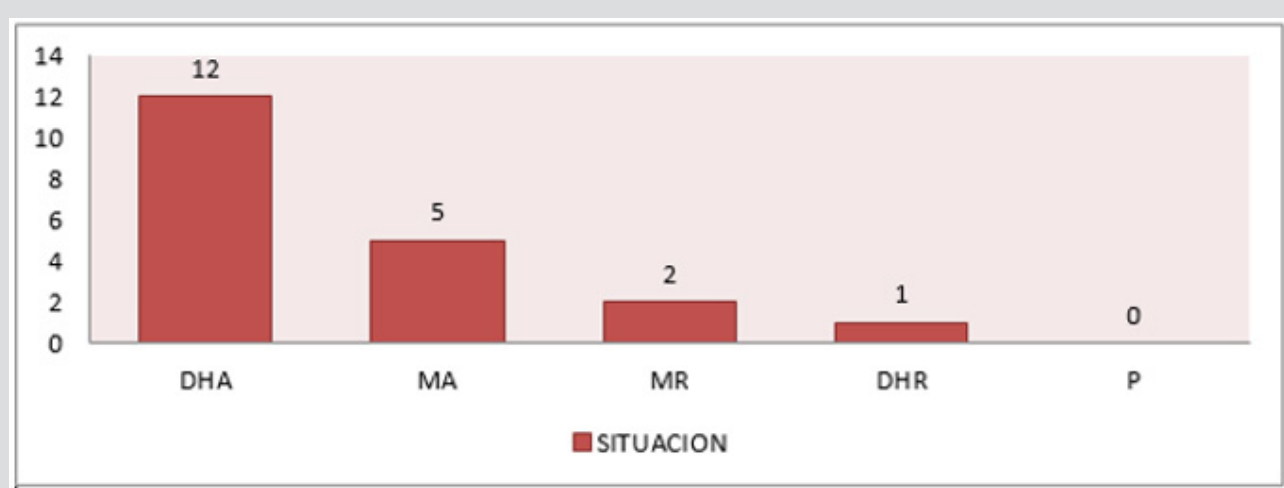

Figure 3: Social security situation.

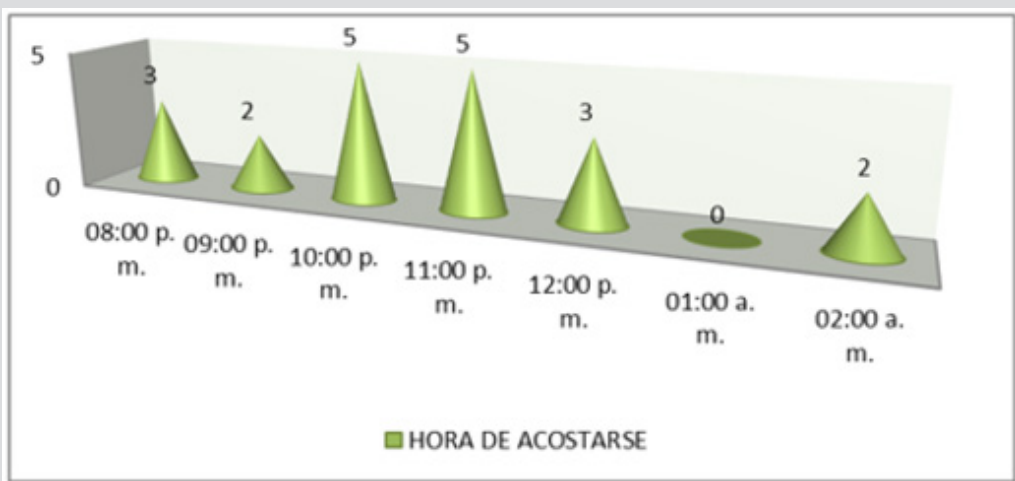

Figure 4: Social security situation.

\section{TIEMPO PARA CONCILIAR EL SUEÑO}

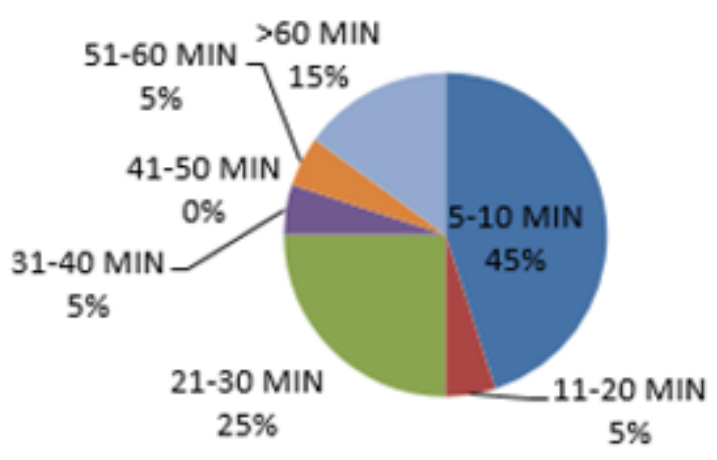

Figure 5: Sleep conciliation time (sleep latency). 


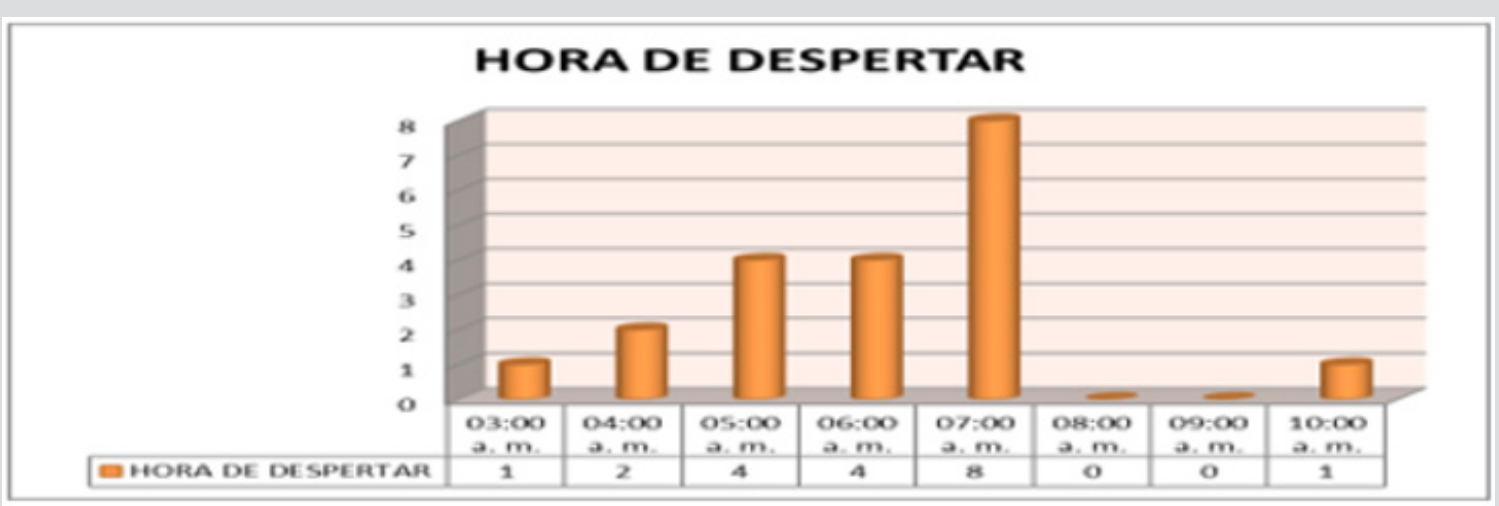

Figure 6: Average wake-up time.

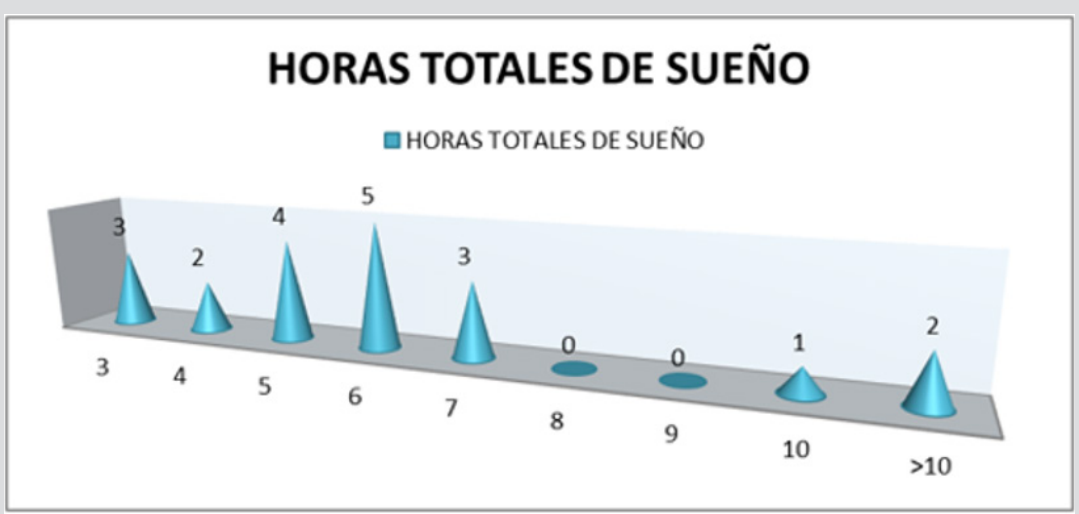

Figure 7.

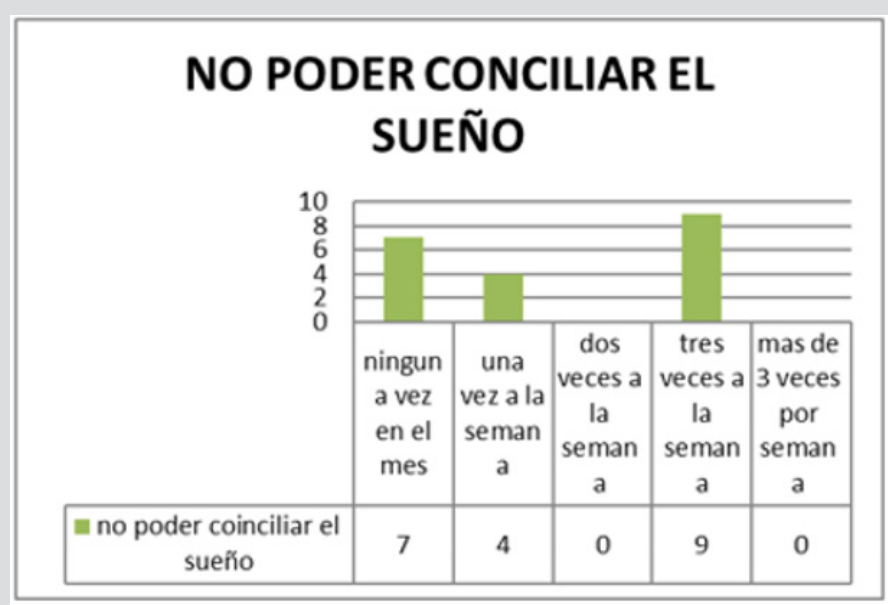

Figure 8.

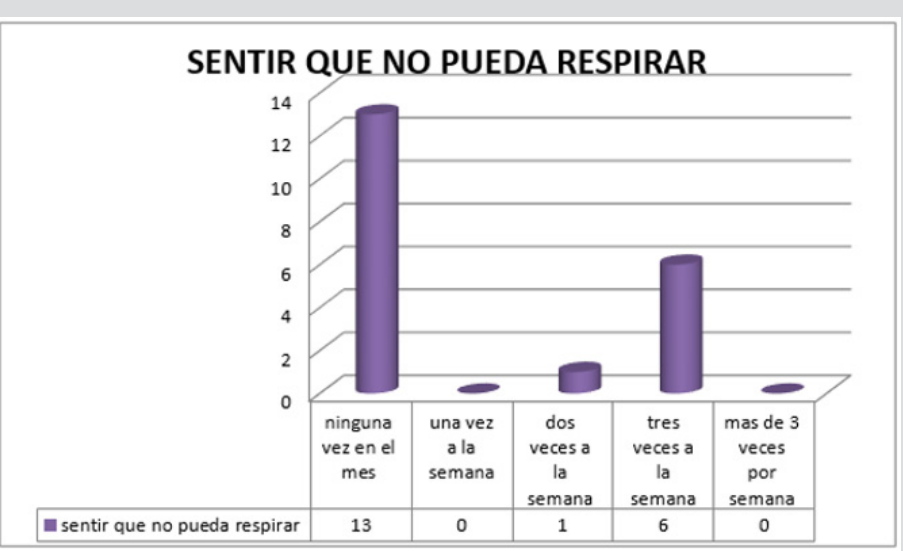

Figure 9. 


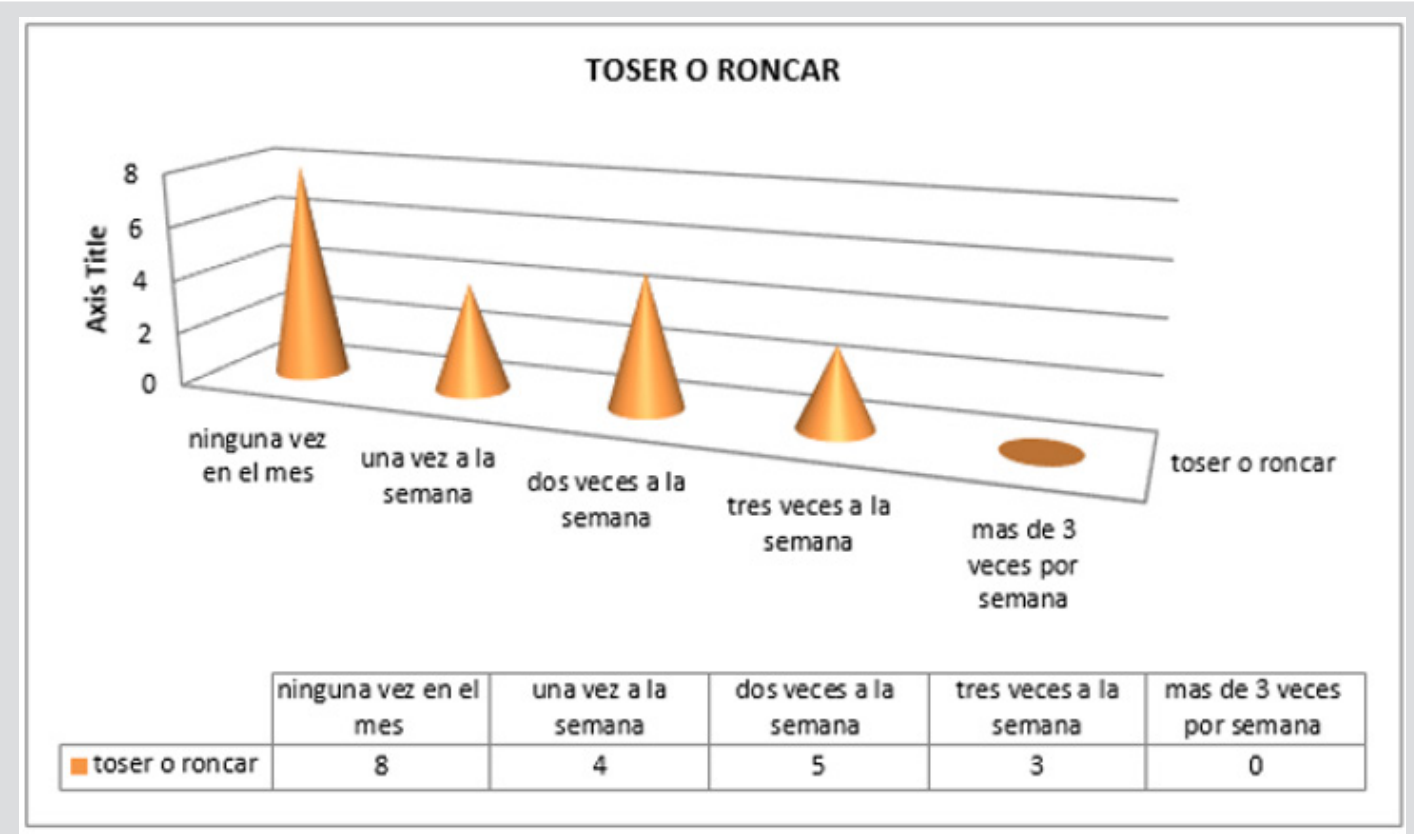

Figure 10.

Continuing with the variability of sleep habits, the question about the total time it takes to sleep is indispensable, where a detailed variability between each point and constant was obtained, obtaining as a result that $45 \%$ of the studied population reconciled sleep within the first 10 minutes, while $25 \%$ is distributed in patients who sleep within the first 30 minutes and an important fact is that $15 \%$ of patient samples last more than 60 minutes to fall asleep (Figure 11). It should be noted the relevance of the time that people take to sleep and the time they have to get up, since it should be noted that within the quality of sleep it is known that a young adult should sleep an average of 7-8 hours, although This amount may vary because it depends on internal factors of the organization and external society, so a preschool child can sleep between 11 or 12 hours and an older adult between 5 and 6 hours not taking into account the periods that doze during the day, so the results are very important in which the percentages of patients who wake up early and sleep late are focused as they begin to have functional and organic damages that are involved in their daily life. Within the Pittsburgh scale it is essential to highlight and highlight the following two patterns:

a) The time when patients regularly wake up from their dreams

b) The total hours of sleep of a patient.

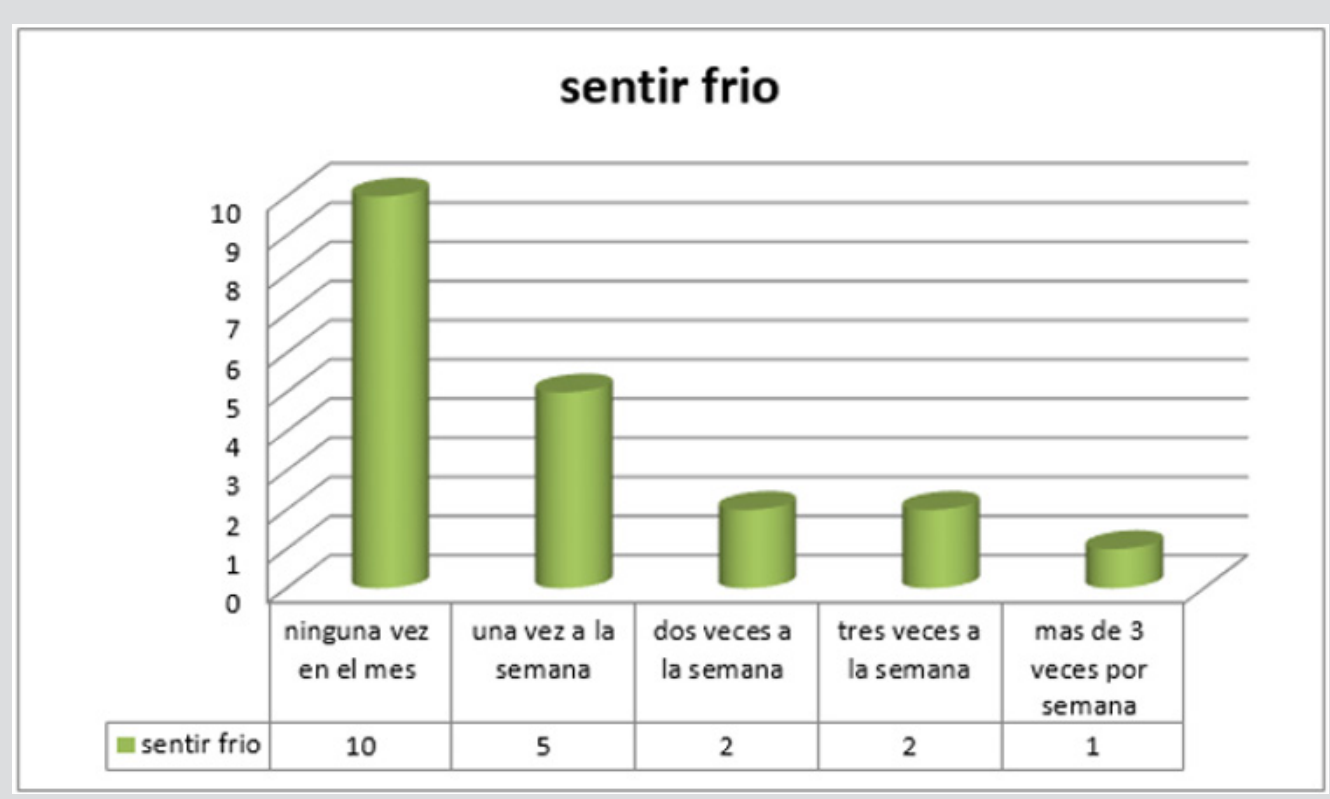

Figure 11. 
Where the results taking as supremacy that $40 \%$ of the studied population wakes up at 7:00 am while the other $40 \%$ wakes up on an average between 5:00 and 6:00 am to perform their activities, finally $15 \%$ It has a short sleep average waking up between 3:00 and 4:00 am (Figures 12-15). Performing and observing an average within the sleep habits and the quality of sleep of the patients a question is asked to evaluate the amount of hours that people sleep approximately, taking into account their basic pathologies (post-operative, chronic pathologies) degenerative, concomitant pathologies), with the result that $45 \%$ of patients only sleep 5 to 6 hours in a row compared to the time a person really has to sleep to refer adequate quality and hygiene of sleep is really low of established parameters, while around $15 \%$ of the patients who were taken as a sample sleep an average between 10 and
15 hours a day, it should be noted that these patients mentioned having psychological and psychiatric problems as depression, if we recapitulate the concepts offered by the national sleep foundation $85 \%$ of the patients interviewed do not meet the criteria to define that they maintain a to adequate sleep health, while only $5 \%$ of patients could enter the criteria already established and mentioned (in the following graph represents the number of patients and the total sleep hours they maintain for a day) the scale of Pittsburgh are observed questions which are worth mentioning that are subjective processes that take relevance in the last 4 weeks, about falling asleep where $45 \%$ of hospitalized patients answered that approximately three times a week they are prevented from falling asleep. a proper way.

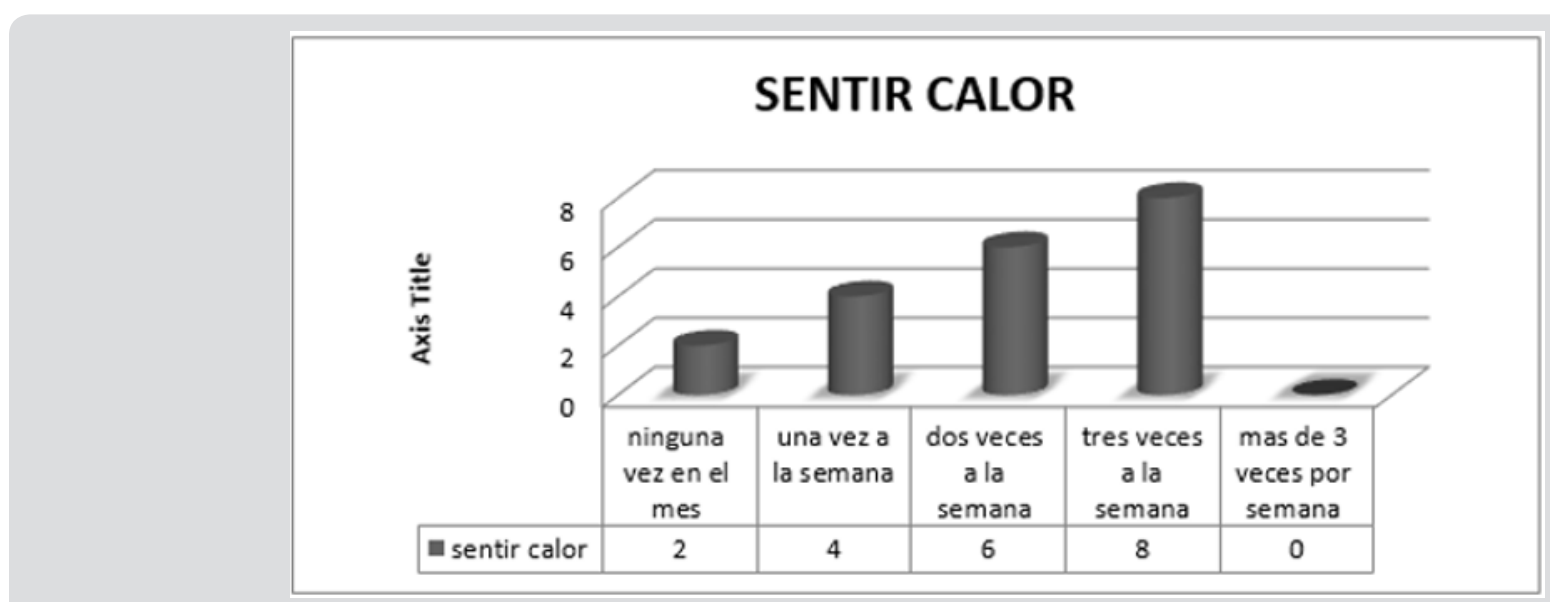

Figure 12.

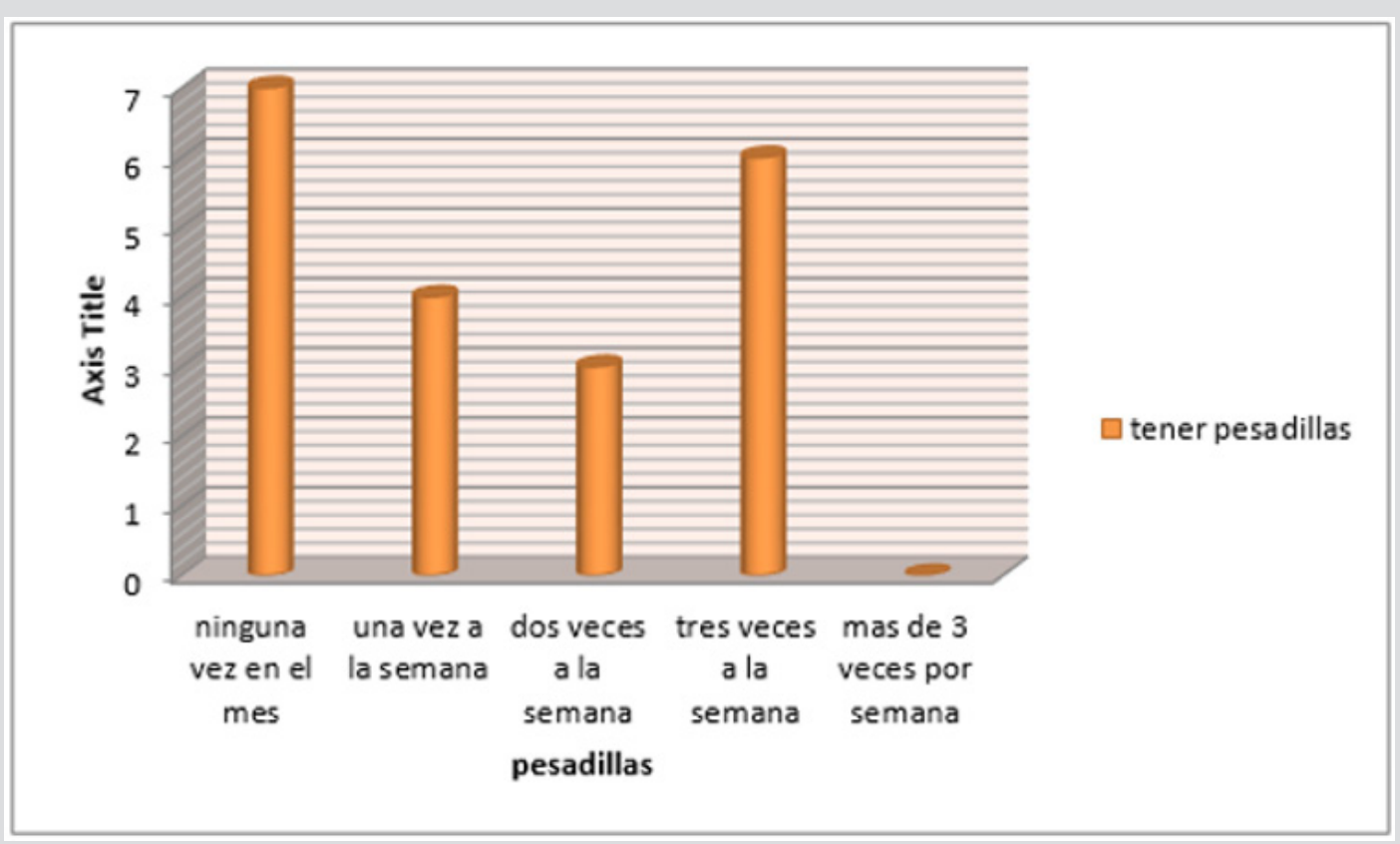

Figure 13. 


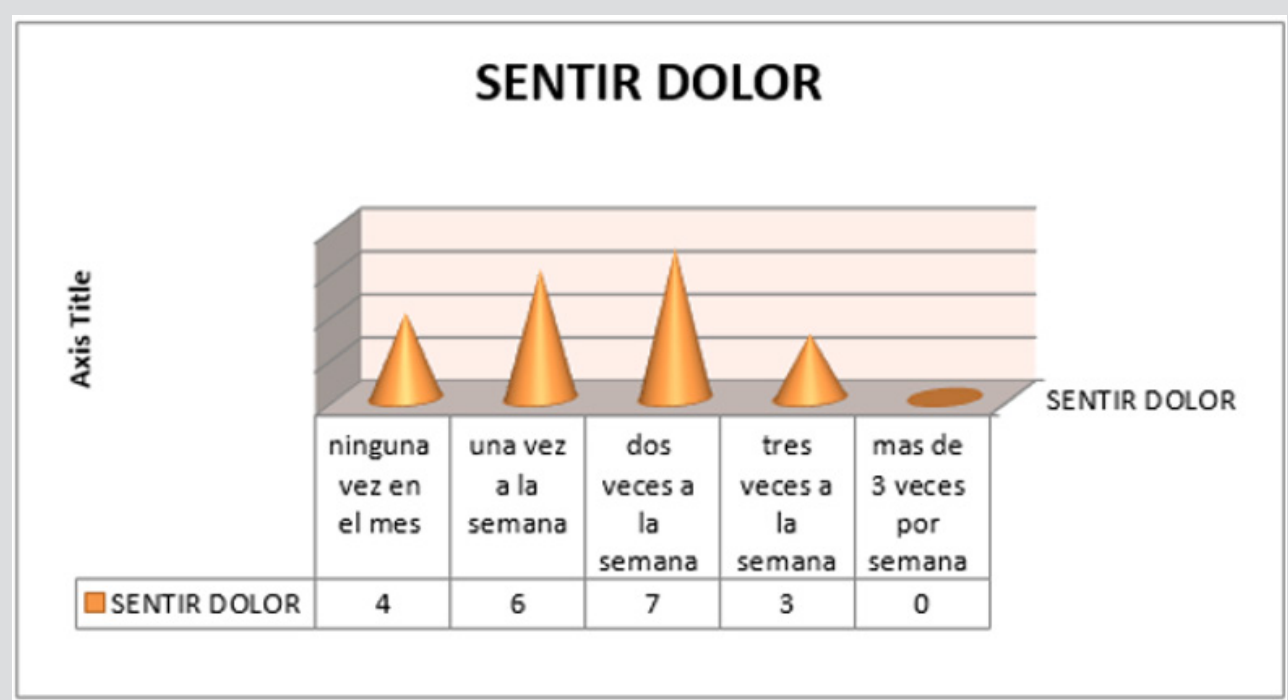

Figure 14.

And evaluating the number of people and how many times they get up during the night, results were obtained that 14 people of the interviewed women get up between 2 and 3 times a week, which is about $70 \%$ of the patients surveyed with those bad sleep habits. Regarding the reasons why patients wake up, the following result was obtained (Figures 15-18). Where $65 \%$ of respondents in the last month would not have suffered from feelings of shortness of breath, while the number of 6 people reported that three times in the week are awakened by feeling a sense of shortness of breath corresponding to a $30 \%$ of the sample investigated.

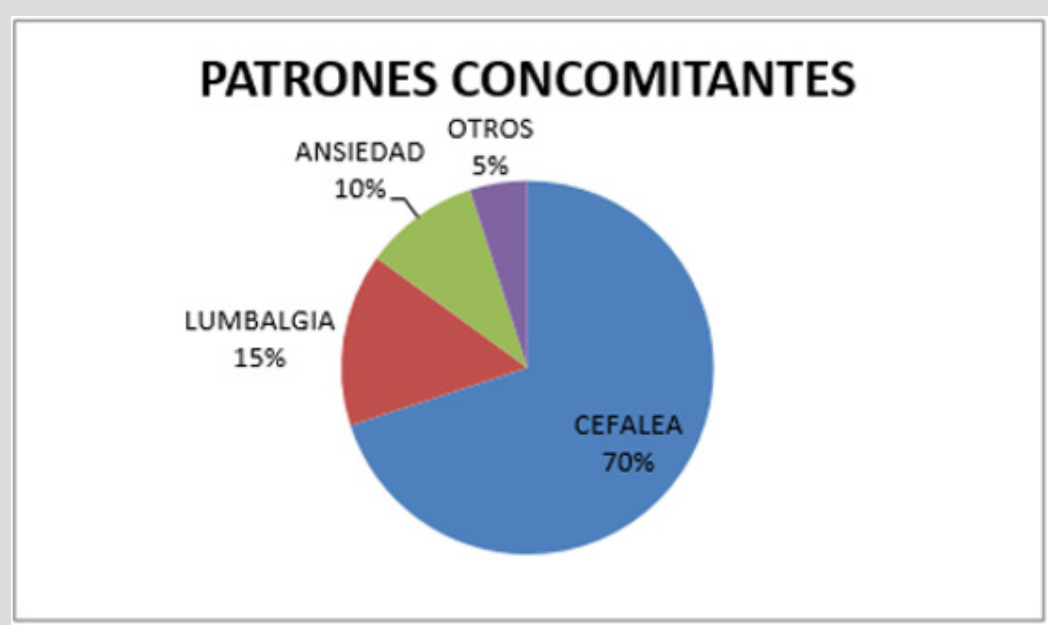

Figure 15.

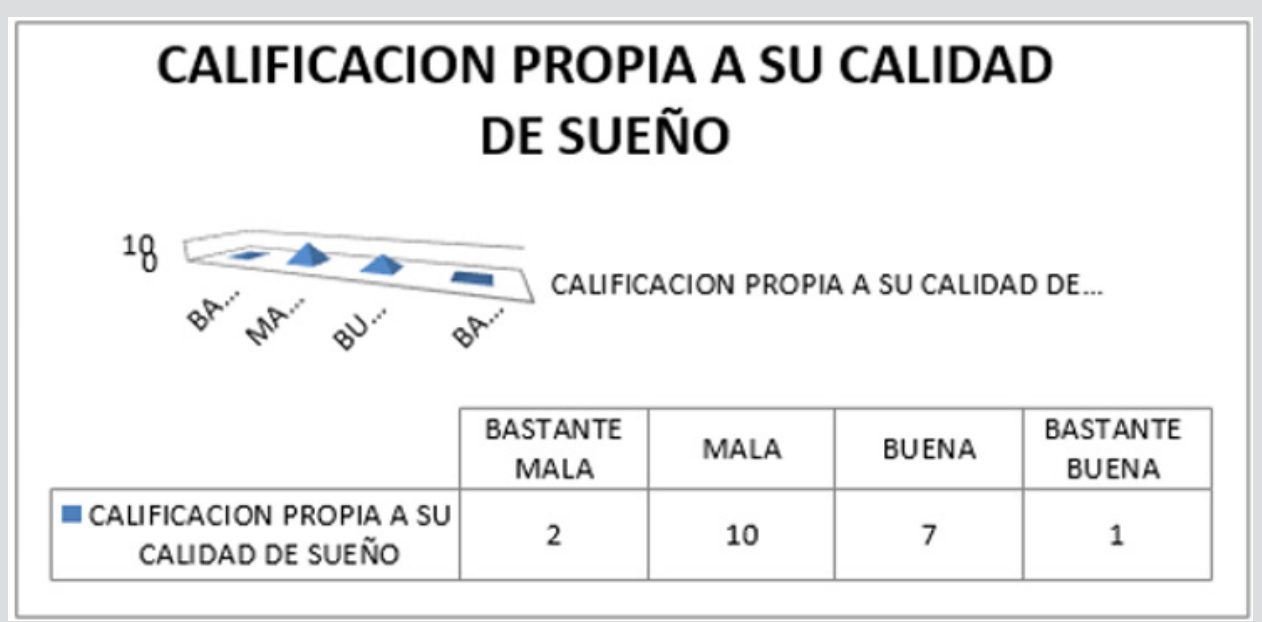

Figure 16. 


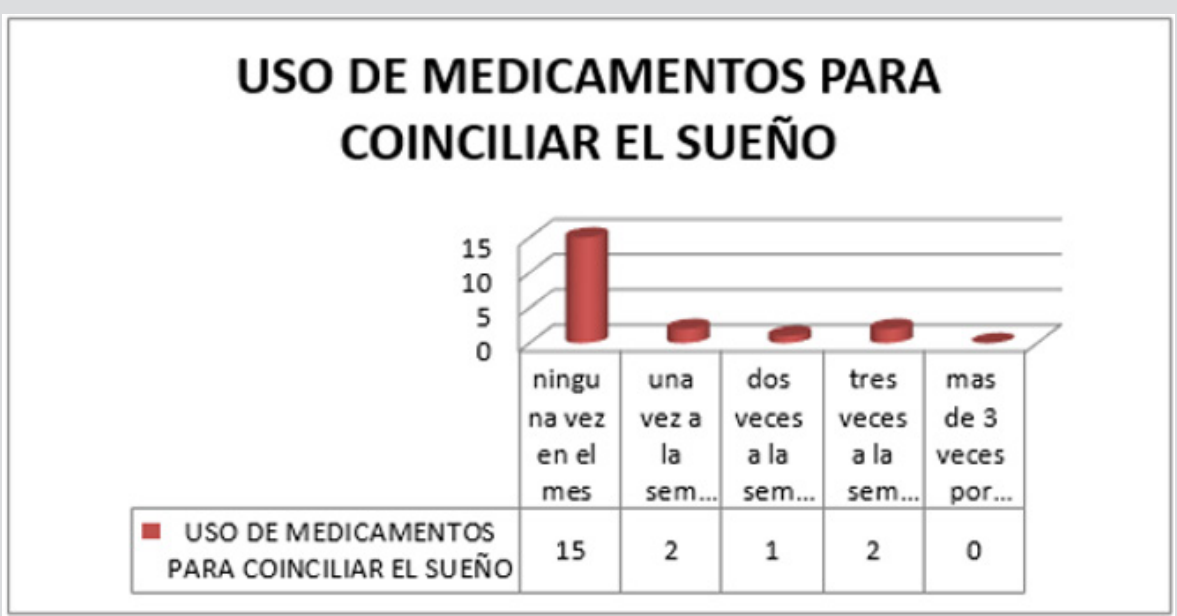

Figure 17.

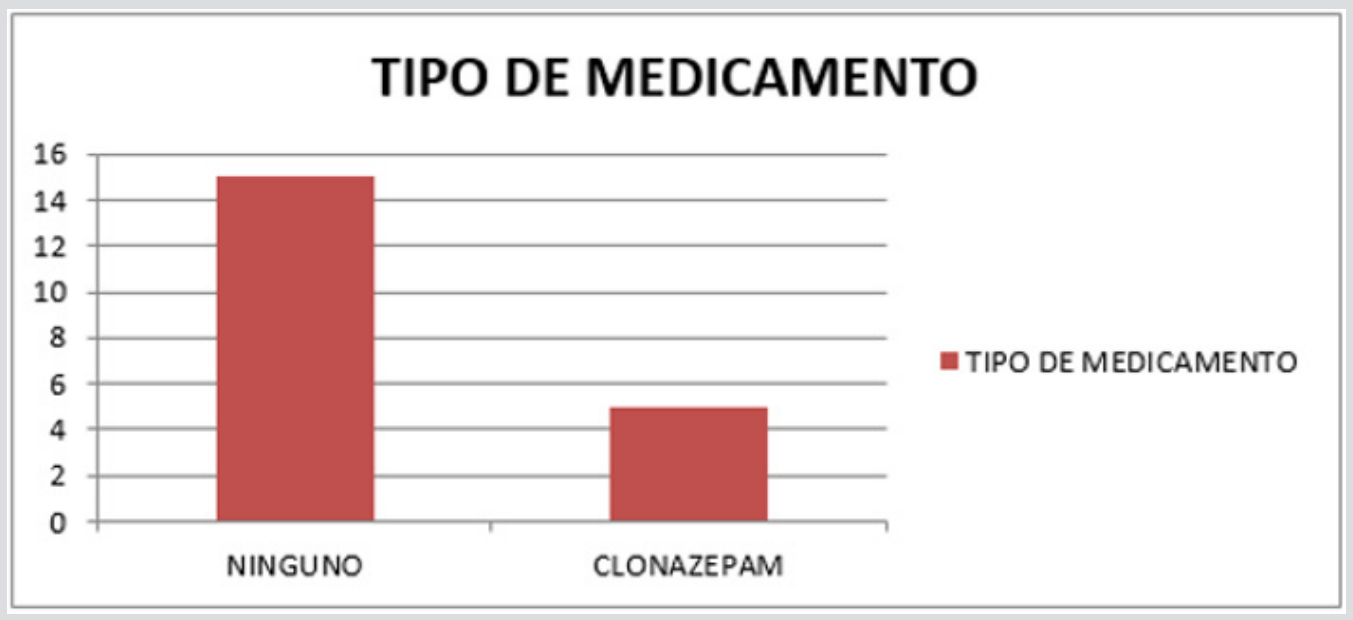

Figure 18.

$\mathrm{l}$ ask indirect questions about if your family member snored $40 \%$ answered that they did not catch such events in the last 4 weeks while $45 \%$ answered that on average 1-2 times per week they snored at night what they did the patient will wake up during the night, $50 \%$ of patients have not woken up during the night due to cold in the last 4 weeks, and with a total of 5 patients it gives a result of $25 \%$ who woke up at least once a week due to cold sensation (Figures $19 \& 20$ ). Otherwise, $70 \%$ of patients used to wake up during the night due to the sensation of heat. $35 \%$ of the patients surveyed had as a result that they have not had problems like nightmares at bedtime, while the other 35\% have nightmares 1 or 2 times a week, $30 \% 3$ times a week; the following graph is representative of the results obtained when applying that question to the sample that was investigated.

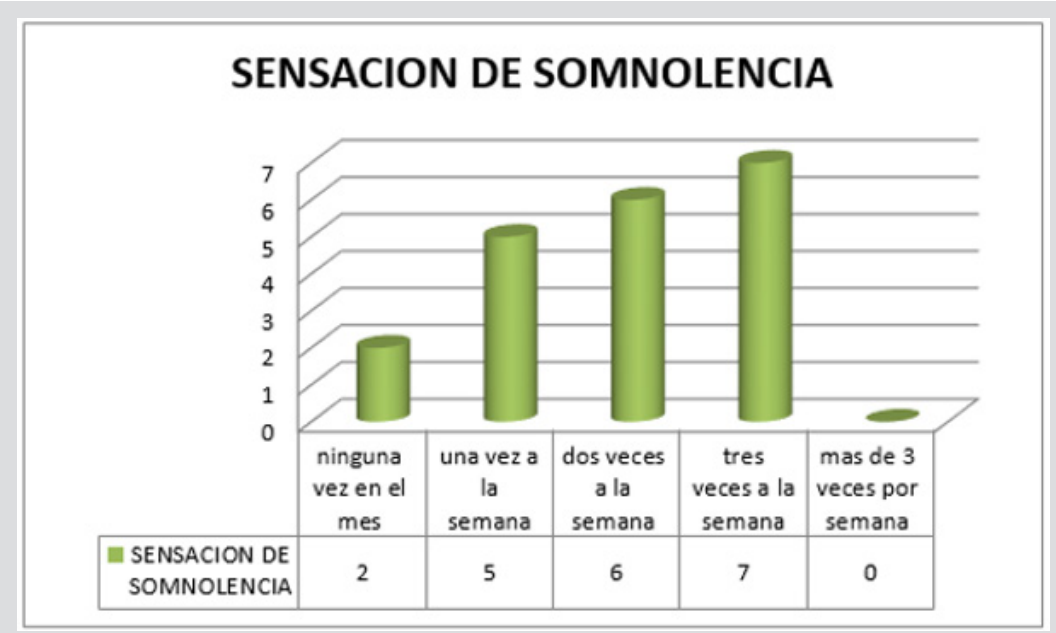

Figure 19. 


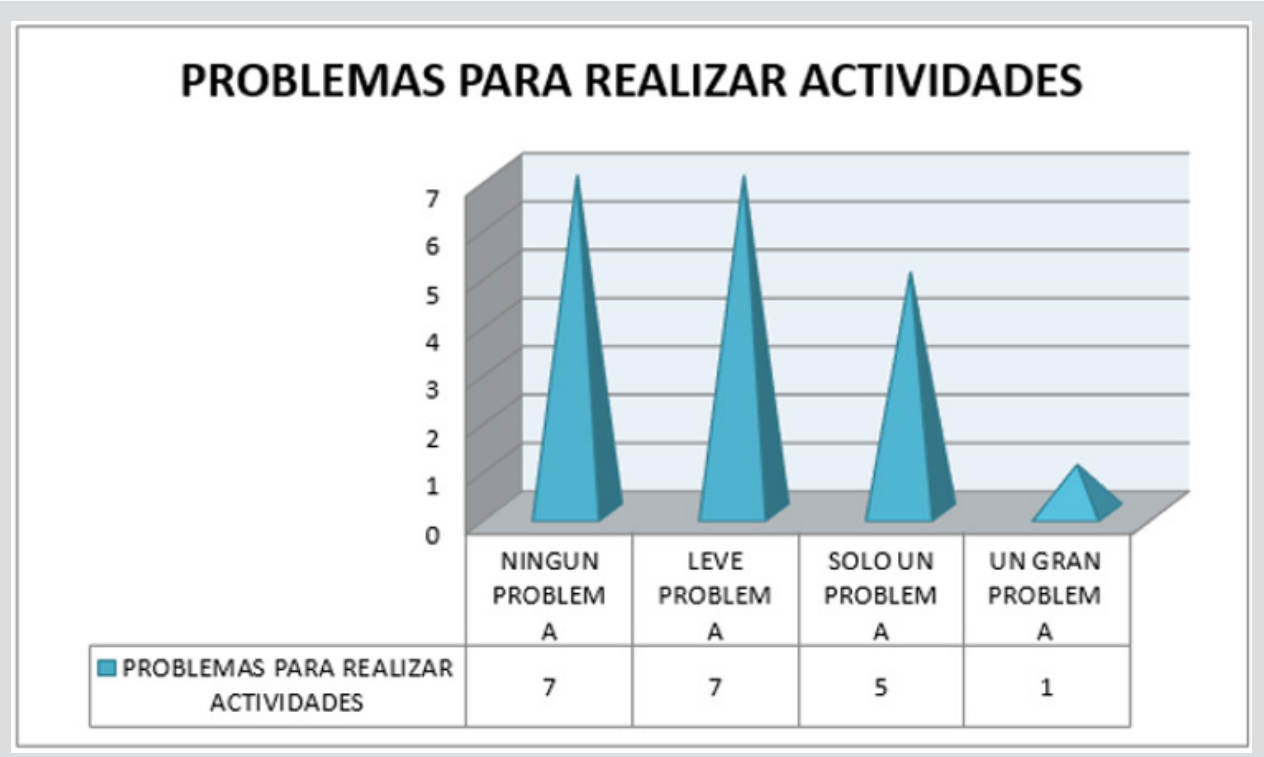

Figure 20.

Among the data that we want to highlight in this study is the presence of pain and of what type in the last 4 weeks, referring to pain as some event that keeps them awake or that awakens them during the night, having as conclusion that the $30 \%$ of the patients interviewed answered affirmatively that approximately once a week felt pain, and 35\% mentioned that approximately twice a week felt some type of pain. Paulatively evaluating the data provided by the patients, it was obtained that $70 \%$ of the hospitalized patients have presented tension headache caused by bad habits or sleep hygiene, adding lumbalgia and even anxiety in a total of 5 more patients that correspond another $25 \%$ more of the sample studied. He was asked if they took any medication or taken in the last 4 weeks to be able to fall asleep, where $75 \%$ denied having taken medication to fall asleep. While the other $25 \%$ agreed to have ingested medication to be able to sleep either 1 to 3 times per week. The unanimous medication that $25 \%$ of patients used is clonazepam which is a benzodiazepine that acts directly on the central nervous system, which can create drowsiness and fatigue.

Within the study of sleep hygiene there is variability where it focuses directly on factors such as drowsiness that is directly caused by poor sleep hygiene habits, based on the Pittsburgh scale there is a direct section where questions about the drowsiness that the patient could suffer and finally if that drowsiness or fatigue could prevent the accomplishment of some daily activity, or executive in the day to day. Having as a result that $65 \%$ of patients have been in a drowsy status or have felt tired in an approximate period of between two times to three times a week, while only $25 \%$ of patients have drowsiness due to at least once a week, preventing their early recovery or decreasing their resistance in everyday life. Taking into account the number of patients who answered affirmatively to the question about sleepiness, a graph was obtained showing the results in which the disability or problem that caused the maintenance of that drowsiness status was shown, obtaining that $35 \%$ of the patients do not have no problem when carrying out their daily activities, while another $35 \%$ of the people surveyed expressed their concern and described as a slight problem in their daily lives the maintenance of somnolence data which can cause difficulties in their daily life; Finally, 25\% classified their level of sleepiness as a moderate problem, defining that in daily activities it is a conflict to be able to perform them with the greatest aptitude and energy.

\section{Conclusion}

Definitely the great problem of health to solve in our country necessarily has to do with habits and because, by not respecting the hours of sleep, biorhythms are altered that result in clinical problems such as: headache, nervous colitis, depression , anxiety, metabolic and systemic problems. It is important to understand the reeducation to our patients in each consultation and sanitary step, since the installation of an adequate diet and weight control, as well as a healthy and aerobic sport together with the sleep hygiene measures will be the main weapons to attack diverse diseases in our times.

\section{References}

1. Lluesma Vidal M, Carmona Simarro JV (2019) Nutritional status regarding the quality of life and sleep pattern in community-dwelling older adults with cognitive dysfunction. 36(2): 303-308.

2. Garrigós Pedrón Me (2019) Predictive factors of sleep quality in patients with chronic migraine. S0213-4853(19): 30014-30013.

3. Serrano Guzmán M, Valenza Peña CM, Serrano-Guzmán C, Aguilar Ferrándiz E, Valenza Demet G (2016) Effects of a dance therapy programme on quality of life, sleep and blood pressure in middle-aged women: A randomised controlled trial. 147(8): 334-339.

4. Abad Massanet Fe (2019) Differences in health-related quality of Life between men and women with sleep-disordered breathing. 41(8): $407-$ 412.

5. Cruz T, García L, Álvarez MA, Manzanero AL (2019) Sleep quality and memory function in healthy ageing. S0213-4853(19): 30003-30009.

6. Escobar Fernández L, Coccolo Góngora A, Vázquez López M, Polo Arrondo AP, Miranda Herrero MC (2019) Continuous spike-waves 
during slow-wave sleep: Experience during 20 years. S1695-4033(19) 30021-30029.

7. Abbott S, Weng J, Reid K, Daviglus M, Gallo L, Loredo J et al. (2019) Sleep Timing, Stability, and BP in the Sueño Ancillary Study of the Hispanic Community Health Study/Study of Latinos. 155(1): 60-68.

8. http://www.paidopsiquiatria.cat/files/trastornos_del_sueno.pdf

ISSN: 2574-1241

DOI: 10.26717/BJSTR.2019.19.003232

Gabriel Miranda Nava. Biomed J Sci \& Tech Res

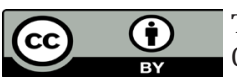

his work is licensed under Creative Commons Attribution 4.0 License

Submission Link: https://biomedres.us/submit-manuscript.php
9. Carrillo Mora P, Ramírez Peris J, Magaña Vázquez K (2019) Neurobiology of the dream and its importance: anthology for the university student.

10. Sierra J, Delgado Domínguez C, Carretero Dios H. Influence of dream quality on psychopathological variables: a comparative analysis among workers submitted to turnos and employees with normal hours. 41(1): 121-130.

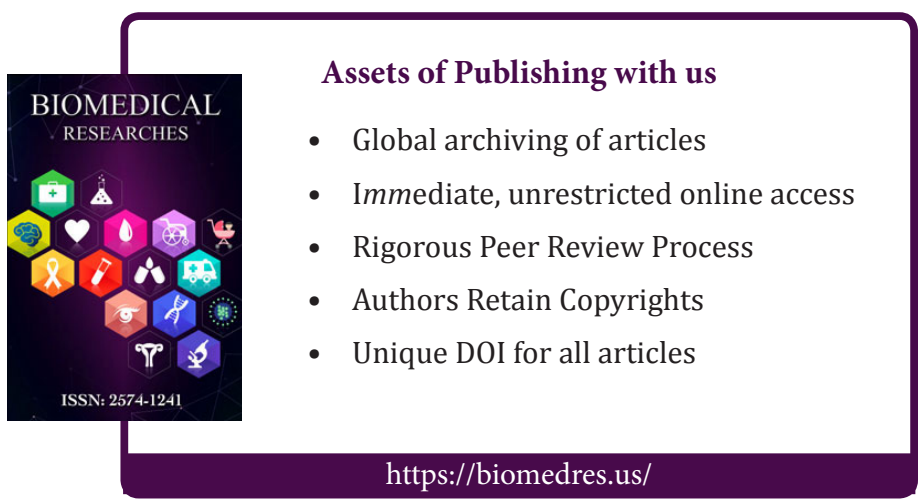

